\title{
A simultaneous Bregman projection methods for solving mixed split equality problems and fixed point problems in p-uniformly convex and uniformly smooth Banach spaces
}

\author{
Haitao Che*, Meixia Li, JingJing Tan \\ School of Mathematics and Information Science, Weifang University, Weifang, 261061, China.
}

Communicated by Y. H. Yao

\begin{abstract}
In this article, a simultaneous Bregman projection scheme is introduced to approximate a common element of the set of fixed points of left Bregman strongly nonexpansive mapping and the set of solutions of mixed split equality problems in $p(p \geqslant 2)$ uniformly convex and uniformly smooth Banach spaces. We obtain the weak convergence theorem of the sequences generated by our scheme under some appropriate conditions. Furthermore, we apply our iterative algorithms to the split feasibility problem. Finally, several numerical results are shown to confirm the feasibility of the proposed methods. Our result presented in the article are new and improve and extend some recent corresponding results. (C)2017 All rights reserved.
\end{abstract}

Keywords: Fixed point, split equality problem, left Bregman strongly nonexpansive mapping, simultaneous iterative method, weak convergence, uniformly convex, uniformly smooth.

2010 MSC: 47H09, 47H05, 47H06, 47J25.

\section{Introduction}

Recently, Moudafi [16] proposed the following split equality problem which is to find

$$
x \in C, y \in Q \text { such that } A x=B y,
$$

where $\mathrm{H}_{1}, \mathrm{H}_{2}$ and $\mathrm{H}_{3}$ are three real Hilbert spaces, $\mathrm{C}$ and $\mathrm{Q}$ are two nonempty closed convex subsets of $\mathrm{H}_{1}$ and $\mathrm{H}_{2}$, respectively, and $A: \mathrm{H}_{1} \rightarrow \mathrm{H}_{3}$ and $\mathrm{B}: \mathrm{H}_{2} \rightarrow \mathrm{H}_{3}$ are two bounded linear operators. The problem (1.1) has been widely studied by many authors [2, 8-10, 12, 15-20, 26, 27], due to its various real-world applications, such as in game theory [2], domain decomposition for PDEs [3], and intensity-modulated radiation therapy (IMRT) [5]. An efficient algorithm for solving the problem (1.1) is the classical projection algorithm which was proposed by Moudafi [16] as follows

$$
\left\{\begin{array}{l}
x_{k+1}=P_{C}\left(x_{k}-\gamma_{k} A^{*}\left(A x_{k}-B y_{k}\right)\right) \\
y_{k+1}=P_{Q}\left(y_{k}+\beta_{k} B^{*}\left(A x_{k}-B y_{k}\right)\right)
\end{array}\right.
$$

where $\gamma_{k}, \beta_{k} \in\left(\varepsilon, \min \left(\frac{1}{\lambda_{A}}, \frac{1}{\lambda_{B}}\right)-\varepsilon\right)$, and $\lambda_{A}$ and $\lambda_{B}$ are the spectral radiuses of $A^{*} A$ and $B^{*} B$, respectively.

\footnotetext{
${ }^{*}$ Corresponding author Tan)

Email addresses: haitaoche@163.com (Haitao Che), limeixia001@163.com (Meixia Li), tanjngjing1108@163.com (JingJing
}

doi:10.22436/jnsa.010.08.38 
He obtained that the sequence $\left(x_{k}, y_{k}\right)$ generated by the algorithm (1.2) converges weakly to a solution of (1.1). In [18], Moudafi proposed the following split common fixed point problem

$$
x \in F(U), y \in F(T) \text { such that } A x=B y,
$$

and introduced the simultaneous algorithm

$$
\left\{\begin{array}{l}
x_{k+1}=U\left(x_{k}-\gamma_{k} A^{*}\left(A x_{k}-B y_{k}\right)\right) \\
y_{k+1}=T\left(y_{k}+\gamma_{k} B^{*}\left(A x_{k}-B y_{k}\right)\right)
\end{array}\right.
$$

for firmly quasi-nonexpansive operators $U$ and $T$, where $\gamma_{k} \in\left(\varepsilon, \frac{2}{\lambda_{A}+\lambda_{B}},-\varepsilon\right)$. The weak convergence of the sequence generated by the simultaneous algorithm (1.4) was investigated.

Observing the algorithms which are mentioned above, we know that the problem (1.1) is treated in the framework of Hilbert spaces. We can find that there is little relevant literature about the convergence of the split equality problem in Banach spaces. Can the problem (1.1) be studied in more general framework, for example, Banach spaces? To obtain it, we first introduce the following mixed split equality problem which is to find

$$
x \in C \cap F(T), y \in Q \cap F(U) \text {, such that } A x=B y,
$$

where $E_{1}, E_{2}$, and $E_{3}$ are Banach spaces. $C \subset E_{1}$ and $Q \subset E_{2}$ are nonempty, closed and convex sets. For $i=1,2,3, E_{i}^{*}$ is the dual space of $E_{i} . A: E_{1} \rightarrow E_{3}$ and $B: E_{2} \rightarrow E_{3}$ are two bounded linear operator, $A^{*}: E_{3}^{*} \rightarrow E_{1}^{*}$ and $B^{*}: E_{3}^{*} \rightarrow E_{2}^{*}$ are the adjoint of $A$ and $B$, respectively. $T$ is a nonlinear mapping of $C$ into $C$, and $U$ is a nonlinear mapping of $Q$ into $Q, F(T)=\{x \in C: x=T x\}$ and $F(U)=\{y \in Q: y=U y\}$. We denote the set of solutions of (1.5) by $\Omega$, i.e.,

$$
\Omega=\{x \in C \cap F(T), y \in Q \cap F(U) \text {, such that } A x=B y\} .
$$

Furthermore, our problem (1.5) extends some recent problems studied by many authors in the literature. Suppose that $T=I$ and $U=I$, where $I$ is the identity map, then $F(T)=C$ and $F(U)=Q$, in this case, our problem (1.5) reduces to the split equality problem (1.1) in the framework of Banach spaces. If $F(T) \subset C$ and $F(U) \subset Q$ for some nonlinear operators $T$ and $U$, then our problem (1.5) reduces to split common fixed point problems (1.3) in the framework of Banach spaces. To solve (1.5), we suggest the following algorithm: for any initial guess $\left(x_{1}, y_{1}\right) \in E_{1} \times E_{2}$, define $\left(x_{n+1}, y_{n+1}\right)$ recursively by the following formula

$$
\left\{\begin{array}{l}
u_{n}=\Pi_{C} J_{E_{1}^{*}}^{q}\left(J_{E_{1}}^{p}\left(x_{n}\right)-\gamma_{n} A^{*} J_{E_{3}}^{p}\left(A x_{n}-B y_{n}\right)\right), \\
x_{n+1}=J_{E_{1}^{*}}^{q}\left(\alpha_{n} J_{E_{1}}^{p}\left(u_{n}\right)+\left(1-\alpha_{n}\right) J_{E_{1}}^{p}\left(T u_{n}\right)\right), \\
v_{n}=\Pi_{Q} J_{E_{2}^{*}}^{q}\left(J_{E_{2}}^{p}\left(y_{n}\right)+\gamma_{n} B^{*} J_{E_{3}}^{p}\left(A x_{n}-B y_{n}\right)\right), \\
y_{n+1}=J_{E_{2}^{*}}^{q}\left(\alpha_{n} J_{E_{2}}^{p}\left(v_{n}\right)+\left(1-\alpha_{n}\right) J_{E_{2}}^{p}\left(u v_{n}\right)\right),
\end{array}\right.
$$

where $\Pi_{C}$ denotes the Bregman projection, $J_{E_{i}^{*}}^{q}$ is the duality mapping of $E_{i}^{*}$, and $J_{E_{i}}^{p}$ is the duality mapping of $E_{i}$ for $i=1,2,3$. Under some appropriate conditions, the weak convergence of the sequence generated by our scheme is investigated.

The article is organized as follows. In Section 2, some definitions and important lemmas are given. In Section 3, we present a simultaneous Bregman projection scheme and establish the weak convergence of the proposed algorithm. We apply our iterative algorithms to the split feasibility problem in Section 4 . In the last section, some simple numerical examples are presented to confirm the feasibility of the proposed methods.

\section{Preliminaries}

We now recall some definitions and lemmas which will be used in the proofs of our main results. 

by

Let $1<q \leqslant 2 \leqslant p$ with $\frac{1}{p}+\frac{1}{q}=1$. The modulus of convexity and smoothness are defined respectively

$$
\delta_{E}(\epsilon)=\inf \left\{1-\frac{\|x+y\|}{2}:\|x\|=\|y\|=1,\|x-y\| \geqslant \epsilon\right\}, \epsilon \in[0,2],
$$

and

$$
\rho_{\mathrm{E}}(\tau)=\left\{\frac{\|x+\tau y\|+\|x-\tau y\|}{2}-1:\|x\|=\|y\|=1\right\}, \tau>0 .
$$

Banach space $E$ is said to be uniformly convex if $\delta_{E}(\epsilon)>0$ for $\epsilon \in(0,2]$, and p-uniformly convex if there exists a constant $C_{q}>0$ such that $\delta_{E}(\epsilon) \geqslant C_{q} \epsilon^{p}$ for $\epsilon \in(0,2]$. Banach space $E$ is said to be uniformly smooth if $\lim _{\tau \rightarrow 0} \frac{\rho_{\mathrm{E}}(\tau)}{\tau}=0$, and q-uniformly smoothness if there exists a constant $\mathrm{C}_{\mathrm{q}}>0$ such that $\rho_{E}(\tau) \geqslant C_{q} \tau^{p}$ for $\tau>0$. The $L_{p}$ space is 2-uniformly convex for $1<p \leqslant 2$ and $p$-uniformly convex for $p \geqslant 2$. From [13], $E$ is $p$-uniformly convex if and only if its dual $E^{*}$ is q-uniformly smooth. As we all know, if $E$ is a $p$-uniformly convex and uniformly smooth, then its dual space $E^{*}$ is $q$-uniformly smooth and uniformly convex. The q-uniformly smooth spaces have the following technical inequality.

Lemma 2.1 ([25]). Let $\mathrm{x}, \mathrm{y} \in \mathrm{E}$. If $\mathrm{E}$ is $q$-uniformly smooth, then there exists a constant $\mathrm{C}_{\mathrm{q}}>0$ such that

$$
\|x-y\|^{q} \leqslant\|x\|^{q}-q\left\langle y, J_{E}^{q}(x)\right\rangle+C_{q}\|y\|^{q} .
$$

The duality mapping $\mathrm{J}_{\mathrm{E}}^{\mathrm{p}}: \mathrm{E} \rightarrow 2^{\mathrm{E}^{*}}$ is defined by

$$
\mathrm{J}_{\mathrm{E}}^{\mathrm{p}}(\mathrm{x})=\left\{\overline{\mathrm{x}} \in \mathrm{E}^{*}:\langle x, \bar{x}\rangle=\|x\|^{\mathrm{p}},\|\overline{\mathrm{x}}\|=\|\mathrm{x}\|^{\mathrm{p}-1}\right\} .
$$

From $[1,11,21]$, the duality mapping $J_{\mathrm{E}}^{\mathrm{p}}$ is one-to-one, single-valued, and has $\mathrm{J}_{\mathrm{E}}^{\mathrm{p}}=\left(\mathrm{J}_{\mathrm{E}^{*}}^{\mathrm{q}}\right)^{-1}$, where $\mathrm{J}_{\mathrm{E}^{*}}^{\mathrm{q}}$ is the duality mapping of $E^{*}$. For any $y \in E$, if

$$
x_{n} \rightarrow x \Rightarrow\left\langle J_{E}^{p}\left(x_{n}\right), y\right\rangle \rightarrow\left\langle J_{E}^{p}(x), y\right\rangle,
$$

then the duality mapping $\mathrm{J}_{\mathrm{E}}^{\mathrm{p}}$ is said to be weak-to-weak continuous.

Assume that $f: E \rightarrow R$ is a Gâteaux differentiable convex function. The function $f$ induces the Bregman distance $\Delta_{\mathrm{f}}(\mathrm{x}, \mathrm{y})$ between $\mathrm{x}, \mathrm{y} \in \mathrm{E}$

$$
\Delta_{f}(x, y)=f(y)-f(x)-\left\langle f^{\prime}(x), y-x\right\rangle, x, y \in E .
$$

From the fact that the duality mapping $J_{E}^{p}$ is the derivative of the function $f_{p}(x)=\frac{1}{p}\|x\|^{p}$, the Bregman distance with respect to $f_{p}$ can be written as

$$
\begin{aligned}
\Delta_{\mathfrak{p}}(x, y)=\frac{1}{\mathrm{q}}\|x\|^{\mathrm{p}}-\left\langle J_{E}^{\mathrm{p}}(x), y\right\rangle+\frac{1}{\mathrm{p}}\|y\|^{\mathrm{p}} & =\frac{1}{\mathrm{p}}\left(\|y\|^{\mathrm{p}}-\|x\|^{\mathrm{p}}\right)+\left\langle J_{E}^{\mathrm{p}}(x), x-y\right\rangle \\
& =\frac{1}{\mathrm{q}}\left(\|x\|^{\mathrm{p}}-\|y\|^{\mathrm{p}}\right)+\left\langle J_{E}^{p}(x)-J_{E}^{\mathrm{p}}(y), x\right\rangle .
\end{aligned}
$$

From [24], for $x, y, z \in E$, one has the following key relations hold

$$
\Delta_{\mathfrak{p}}(x, y)=\Delta_{\mathfrak{p}}(x, z)+\Delta_{\mathfrak{p}}(z, y)+\left\langle z-y, J_{\mathrm{E}}^{\mathrm{p}}(x)-\mathrm{J}_{\mathrm{E}}^{\mathrm{p}}(z)\right\rangle,
$$

and, for a constant $\tau>0$,

$$
\tau\|x-y\|^{p} \leqslant \Delta_{p}(x, y) \leqslant\left\langle x-y, J_{E}^{p}(x)-J_{E}^{p}(y)\right\rangle .
$$

Let $C$ be a nonempty, closed, and convex subset of $E$. The metric projection of $x \in X$ onto $C$ is the unique element $P_{C} x \in C$ such that

$$
P_{C} x=\arg \min _{y \in C}\|x-y\|, x \in E,
$$


which leads to the following variational inequality

$$
\left\langle J_{\mathrm{E}}^{\mathrm{p}}\left(x-\mathrm{P}_{\mathrm{C}} x\right), z-\mathrm{P}_{\mathrm{C}} x\right\rangle \leqslant 0, \quad \forall z \in \mathrm{C} .
$$

Similar to the metric projection, Bregman projections are defined as minimizers of Bregman distances [23]. The Bregman projection can be defined by

$$
\Pi_{C} x=\arg \min _{y \in C} \Delta_{p}(x, y), x \in E,
$$

which can also be rewritten as the following variational inequality

$$
\left\langle\mathrm{J}_{\mathrm{E}}^{\mathrm{p}}(\mathrm{x})-\mathrm{J}_{\mathrm{E}}^{\mathrm{p}}\left(\Pi_{\mathrm{C}} \mathrm{x}\right), z-\Pi_{\mathrm{C}} \mathrm{x}\right\rangle \leqslant 0, \forall z \in \mathrm{C} .
$$

Furthermore,

$$
\Delta_{\mathfrak{p}}\left(\Pi_{\mathrm{C}} x, z\right) \leqslant \Delta_{\mathfrak{p}}(x, z)-\Delta_{\mathfrak{p}}\left(x, \Pi_{\mathrm{C}} x\right), \forall z \in \mathrm{C} .
$$

Moreover, for any $z \in E,\left\{x_{i}\right\}_{i=1}^{N} \in E$ and $\left\{t_{i}\right\}_{i=1}^{N} \in(0,1)$ with $\sum_{i=1}^{n} t_{i}=1$, the inequality

$$
\Delta_{\mathrm{p}}\left(\mathrm{J}_{\mathrm{E}^{*}}^{\mathrm{q}}\left(\sum_{i=1}^{\mathrm{N}} \mathrm{t}_{\mathrm{i}} \mathrm{J}_{\mathrm{E}}^{\mathrm{p}}\left(\mathrm{x}_{\mathrm{i}}\right)\right), z\right) \leqslant \sum_{i=1}^{\mathrm{N}} \mathrm{t}_{i} \Delta_{\mathrm{p}}\left(\mathrm{x}_{i}, z\right)
$$

is valid. In Hilbert spaces, the metric projection and the Bregman projection are coincident with respect to $f_{2}$, but the metric projection can not share the property (2.3) as the Bregman projection in Banach spaces.

Let $T$ be a self-mapping of $C$. If a sequence $\left\{x_{n}\right\}_{n=1}^{\infty} \in C$ converges weakly to $p \in C$ and $\lim _{n \rightarrow \infty} \| x_{n}-$ $T x_{n} \|=0$, the point $p$ is called an asymptotic fixed point $[7,22]$ of $T . \widehat{F}(T)$ denotes the set of asymptotic fixed points of T. Following [14, 22, 27], the definition of left Bregman strongly nonexpansive is shown.

Definition 2.2. A nonlinear mapping $T$ with a nonempty asymptotic fixed point set is said to be left Bregman strongly nonexpansive with respect to a nonempty $\widehat{F}(T)$ if

$$
\Delta_{\mathfrak{p}}(\mathrm{T} x, \bar{x}) \leqslant \Delta_{\mathfrak{p}}(x, \bar{x}), \quad \forall x \in \mathrm{C}, \bar{x} \in \widehat{\mathrm{F}}(\mathrm{T})
$$

and if $\left\{x_{n}\right\} \subset C$ is bounded, $\bar{x} \in \widehat{F}(T)$, and

$$
\lim _{n \rightarrow \infty}\left(\Delta_{\mathfrak{p}}\left(x_{n}, \bar{x}\right)-\Delta_{p}\left(T x_{n}, \bar{x}\right)\right)=0,
$$

it follows that

$$
\lim _{n \rightarrow \infty} \Delta_{p}\left(x_{n}, T x_{n}\right)=0 .
$$

Throughout this paper, let $1<q \leqslant 2 \leqslant p<\infty$ with $\frac{1}{p}+\frac{1}{q}=1$. Assume that $E_{1}, E_{2}$, and $E_{3}$ are p-uniformly convex and uniformly smooth Banach spaces. We further denote by $J_{E_{1}}^{p} J_{E_{2}}^{p}$ and $J_{E_{3}}^{p}$ the duality mappings of $E_{1}, E_{2}$, and $E_{3}$, respectively. We apply $\rightarrow$ to denote the weak convergence, and use $\omega_{w}\left(x_{k}\right)=\left\{x: \exists x_{k_{j}} \rightarrow x\right\}$ to express the weak $\omega$-limit set of $\left\{x_{k}\right\}$.

\section{Simultaneous Bregman projection scheme for the problem (1.5)}

In this section, we introduce a simultaneous Bregman projection scheme to approximate a common element of the set of fixed points of left Bregman strongly nonexpansive mapping and the set of solutions of split equality problems in p-uniformly convex and uniformly smooth Banach spaces. We obtain the weak convergence theorem of the sequences generated by our scheme under some appropriate conditions.

Now, we are in a position to show our main results. 
Theorem 3.1. Let $E_{1}, E_{2}$, and $E_{3}$ be three p-uniformly convex and uniformly smooth real Banach spaces. Let $\mathrm{A}: \mathrm{E}_{1} \rightarrow \mathrm{E}_{3}$ and $\mathrm{B}: \mathrm{E}_{2} \rightarrow \mathrm{E}_{3}$ be two bounded linear operators, $\mathrm{A}^{*}: \mathrm{E}_{3}^{*} \rightarrow \mathrm{E}_{1}^{*}$ and $\mathrm{B}^{*}: \mathrm{E}_{3}^{*} \rightarrow \mathrm{E}_{2}^{*}$ be the adjoints of $\mathrm{A}$ and $\mathrm{B}$, respectively. Let $\mathrm{T}$ be a left Bregman strongly nonexpansive mapping of $\mathrm{C}$ into $\mathrm{C}$ such that $\mathrm{F}(\mathrm{T})=\hat{\mathrm{F}}(\mathrm{T})$, and $\mathrm{U}$ be a left Bregman strongly nonexpansive mapping of $\mathrm{Q}$ into $\mathrm{Q}$ such that $\mathrm{F}(\mathrm{U})=\hat{\mathrm{F}}(\mathrm{U})$, where $\mathrm{C} \subset \mathrm{E}_{1}$ and $\mathrm{Q} \subset \mathrm{E}_{2}$ are nonempty, closed, and convex sets. For any initial guess $\left(\mathrm{x}_{1}, \mathrm{y}_{1}\right) \in \mathrm{E}_{1} \times \mathrm{E}_{2}$, define $\left(x_{n+1}, y_{n+1}\right)$ recursively by (1.6). Suppose that $\left\{\alpha_{n}\right\}$ is a sequence in $(0,1)$ and the sequence $\left\{\gamma_{n}\right\}$ satisfies $0<\mathrm{a}_{1}<\gamma_{\mathrm{n}}<\mathrm{a}_{2}<\left(\frac{\mathrm{q}}{\mathrm{C}_{\mathrm{q}}\left(\|\mathrm{A}\|^{\mathrm{q}}+\|\mathrm{B}\| \mathrm{q}\right)}\right)^{\frac{1}{q-1}}$. If the solution set $\Omega$ of (1.5) is nonempty, then the sequence $\left(x_{n}, y_{n}\right)$ generated by (1.6) converges weakly to a solution of (1.5). Furthermore, $\left\|A x_{n}-B y_{n}\right\| \rightarrow 0,\left\|x_{n}-x_{n+1}\right\| \rightarrow 0$, and $\left\|\mathrm{y}_{\mathrm{n}}-\mathrm{y}_{\mathrm{n}+1}\right\| \rightarrow 0$ as $\mathrm{n} \rightarrow \infty$.

Proof. Assume that $\left(x^{*}, y^{*}\right) \in \Omega$, then $A x^{*}=B y^{*}$. Set $t_{n}=J_{E_{1}^{*}}^{q}\left(J_{E_{1}}^{p}\left(x_{n}\right)-\gamma_{n} A^{*} J_{E_{3}}^{p}\left(A x_{n}-B y_{n}\right)\right)$ and $w_{n}=J_{E_{2}^{*}}^{q}\left(J_{E_{2}}^{p}\left(y_{n}\right)+\gamma_{n} B^{*} J_{E_{3}}^{p}\left(A x_{n}-B y_{n}\right)\right)$. It follows from (1.6) and Lemma 2.1 that

$$
\begin{aligned}
\Delta_{p}\left(u_{n}, x^{*}\right)= & \Delta_{p}\left(\Pi_{C} t_{n}, x^{*}\right) \\
\leqslant & \Delta_{p}\left(t_{n}, x^{*}\right) \\
= & \frac{1}{q}\left\|t_{n}\right\|^{p}-\left\langle J_{E_{1}}^{p} t_{n}, x^{*}\right\rangle+\frac{1}{p}\left\|x^{*}\right\|^{p} \\
= & \frac{1}{q}\left\|J_{E_{1}}^{p}\left(x_{n}\right)-\gamma_{n} A^{*} J_{E_{3}}^{p}\left(A x_{n}-B y_{n}\right)\right\|^{q}-\left\langle J_{E_{1}}^{p}\left(x_{n}\right), x^{*}\right\rangle \\
& +\gamma_{n}\left\langle A^{*} J_{E_{3}}^{p}\left(A x_{n}-B y_{n}\right), x^{*}\right\rangle+\frac{1}{p}\left\|x^{*}\right\|^{p} \\
\leqslant & \frac{1}{q}\left\|J_{E_{1}}^{p}\left(x_{n}\right)\right\|^{q}-\gamma_{n}\left\langle A^{*} J_{E_{3}}^{p}\left(A x_{n}-B y_{n}\right), x_{n}\right\rangle+\frac{C_{q}\left(\gamma_{n}\|A\|\right)^{q}}{q}\left\|J_{E_{3}}^{p}\left(A x_{n}-B y_{n}\right)\right\|^{q} \\
& -\left\langle J_{E_{1}}^{p}\left(x_{n}\right), x^{*}\right\rangle+\gamma_{n}\left\langle A^{*} J_{E_{3}}^{p}\left(A x_{n}-B y_{n}\right), x^{*}\right\rangle+\frac{1}{p}\left\|x^{*}\right\|^{p} \\
= & \frac{1}{q}\left\|x_{n}\right\|^{p}-\left\langle J_{E_{1}}^{p}\left(x_{n}\right), x^{*}\right\rangle+\frac{1}{p}\left\|x^{*}\right\|^{p}-\gamma_{n}\left\langle J_{E_{3}}^{p}\left(A x_{n}-B y_{n}\right), A x_{n}-A x^{*}\right\rangle \\
& +\frac{C_{q}\left(\gamma_{n}\|A\|\right)^{q}}{q}\left\|J_{E_{3}}^{p}\left(A x_{n}-B y_{n}\right)\right\|^{q} \\
= & \Delta_{p}\left(x_{n}, x^{*}\right)-\gamma_{n}\left\langle J_{E_{3}}^{p}\left(A x_{n}-B y_{n}\right), A x_{n}-A x^{*}\right\rangle+\frac{C_{q}\left(\gamma_{n}\|A\|\right)^{q}}{q}\left\|A x_{n}-B y_{n}\right\|^{p}
\end{aligned}
$$

and

$$
\begin{aligned}
& \Delta_{\mathfrak{p}}\left(v_{n}, y^{*}\right)=\Delta_{\mathfrak{p}}\left(\Pi_{C} w_{n}, y^{*}\right) \\
& \leqslant \Delta_{\mathrm{p}}\left(w_{n}, \mathrm{y}^{*}\right) \\
& =\frac{1}{\mathrm{q}}\left\|w_{n}\right\|^{\mathrm{p}}-\left\langle\mathrm{J}_{\mathrm{E}_{2}}^{\mathrm{p}} w_{n}, x^{*}\right\rangle+\frac{1}{\mathrm{p}}\left\|x^{*}\right\|^{\mathrm{p}} \\
& =\frac{1}{q}\left\|J_{E_{2}}^{p}\left(y_{n}\right)+\gamma_{n} B^{*} J_{E_{3}}^{p}\left(A x_{n}-B y_{n}\right)\right\|^{q}-\left\langle J_{E_{2}}^{p}\left(y_{n}\right), y^{*}\right\rangle \\
& -\gamma_{n}\left\langle B^{*} J_{E_{3}}^{p}\left(A x_{n}-B y_{n}\right), y^{*}\right\rangle+\frac{1}{p}\left\|y^{*}\right\|^{p} \\
& \leqslant \frac{1}{q}\left\|J_{E_{1}}^{p}\left(y_{n}\right)\right\|^{q}+\gamma_{n}\left\langle B^{*} J_{E_{3}}^{p}\left(A x_{n}-B y_{n}\right), y_{n}\right\rangle+\frac{C_{q}\left(\gamma_{n}\|B\|\right)^{q}}{q}\left\|J_{E_{3}}^{p}\left(A x_{n}-B y_{n}\right)\right\|^{q} \\
& -\left\langle J_{E_{2}}^{p}\left(y_{n}\right), y^{*}\right\rangle-\gamma_{n}\left\langle B^{*} J_{E_{3}}^{p}\left(A x_{n}-B y_{n}\right), y^{*}\right\rangle+\frac{1}{p}\left\|y^{*}\right\|^{p} \\
& =\frac{1}{q}\left\|y_{n}\right\|^{p}-\left\langle J_{E_{2}}^{p}\left(y_{n}\right), y^{*}\right\rangle+\frac{1}{p}\left\|y^{*}\right\|^{p}-\gamma_{n}\left\langle J_{E_{3}}^{p}\left(A x_{n}-B y_{n}\right), B y^{*}-B y_{n}\right\rangle
\end{aligned}
$$




$$
\begin{aligned}
& +\frac{C_{q}\left(\gamma_{n}\|B\|\right)^{q}}{q}\left\|J_{E_{3}}^{p}\left(A x_{n}-B y_{n}\right)\right\|^{q} \\
= & \Delta_{p}\left(y_{n}, y^{*}\right)-\gamma_{n}\left\langle J_{E_{3}}^{p}\left(A x_{n}-B y_{n}\right), B y^{*}-B y_{n}\right\rangle+\frac{C_{q}\left(\gamma_{n}\|B\|\right)^{q}}{q}\left\|A x_{n}-B y_{n}\right\|^{p} .
\end{aligned}
$$

Furthermore, (1.6) and (2.4) reveal that

$$
\begin{aligned}
\Delta_{\mathfrak{p}}\left(x_{n+1}, x^{*}\right) & =\Delta_{\mathfrak{p}}\left(\mathrm{J}_{\mathrm{E}_{1}^{*}}^{\mathrm{q}}\left(\alpha_{n} J_{\mathrm{E}_{1}}^{\mathrm{p}}\left(u_{n}\right)+\left(1-\alpha_{n}\right) J_{\mathrm{E}_{1}}^{\mathrm{p}}\left(T u_{n}\right)\right), x^{*}\right) \\
& \leqslant \alpha_{n} \Delta_{\mathfrak{p}}\left(u_{n}, x^{*}\right)+\left(1-\alpha_{n}\right) \Delta_{\mathfrak{p}}\left(T u_{n}, x^{*}\right) \\
& \leqslant \alpha_{n} \Delta_{\mathfrak{p}}\left(u_{n}, x^{*}\right)+\left(1-\alpha_{n}\right) \Delta_{\mathfrak{p}}\left(u_{n}, x^{*}\right) \\
& \leqslant \Delta_{\mathfrak{p}}\left(u_{n}, x^{*}\right)
\end{aligned}
$$

and

$$
\begin{aligned}
\Delta_{\mathfrak{p}}\left(y_{n+1}, y^{*}\right) & =\Delta_{\mathfrak{p}}\left(J_{E_{2}^{*}}^{q}\left(\alpha_{n} J_{E_{2}}^{\mathrm{p}}\left(v_{n}\right)+\left(1-\alpha_{n}\right) J_{\mathrm{E}_{2}}^{\mathrm{p}}\left(\mathrm{U} v_{n}\right)\right), \mathrm{y}^{*}\right) \\
& \leqslant \alpha_{n} \Delta_{\mathfrak{p}}\left(v_{n}, \mathrm{y}^{*}\right)+\left(1-\alpha_{n}\right) \Delta_{\mathfrak{p}}\left(\mathrm{U} v_{n}, \mathrm{y}^{*}\right) \\
& \leqslant \alpha_{n} \Delta_{\mathfrak{p}}\left(v_{n}, \mathrm{y}^{*}\right)+\left(1-\alpha_{n}\right) \Delta_{\mathfrak{p}}\left(v_{n}, \mathrm{y}^{*}\right) \\
& \leqslant \Delta_{\mathrm{p}}\left(v_{n}, \mathrm{y}^{*}\right) .
\end{aligned}
$$

Adding the above two inequalities, using (3.1), (3.2), and the fact $A x^{*}=B y^{*}$, one has

$$
\begin{aligned}
\Delta_{p}\left(x_{n+1}, x^{*}\right)+\Delta_{p}\left(y_{n+1}, y^{*}\right) \leqslant & \Delta_{p}\left(u_{n}, x^{*}\right)+\Delta_{p}\left(v_{n}, y^{*}\right) \\
\leqslant & \Delta_{p}\left(x_{n}, x^{*}\right)+\Delta_{p}\left(y_{n}, x^{*}\right)-\gamma_{n}\left\langle J_{E_{3}}^{p}\left(A x_{n}-B y_{n}\right), A x_{n}-B y_{n}\right\rangle \\
& +\frac{C_{q}\left(\left(\gamma_{n}\|A\|\right)^{q}+\left(\gamma_{n}\|B\|\right)^{q}\right)}{q}\left\|A x_{n}-B y_{n}\right\|^{p} \\
= & \Delta_{p}\left(x_{n}, x^{*}\right)+\Delta_{p}\left(y_{n}, y^{*}\right) \\
& -\left(\gamma_{n}-\frac{C_{q}\left(\left(\gamma_{n}\|A\|\right)^{q}+\left(\gamma_{n}\|B\|\right)^{q}\right)}{q}\right)\left\|A x_{n}-B y_{n}\right\|^{p} .
\end{aligned}
$$

Now, setting $\Gamma_{n}\left(x^{*}, y^{*}\right)=\Delta_{p}\left(x_{n}, x^{*}\right)+\Delta_{p}\left(y_{n}, y^{*}\right)$, we can write (3.5) to the following key inequality

$$
\Gamma_{n+1}\left(x^{*}, y^{*}\right) \leqslant \Gamma_{n}\left(x^{*}, y^{*}\right)-\left(\gamma_{n}-\frac{C_{q}\left(\left(\gamma_{n}\|A\|\right)^{q}+\left(\gamma_{n}\|B\|\right)^{q}\right)}{q}\right)\left\|A x_{n}-B y_{n}\right\|^{p},
$$

which with the condition of $\gamma_{n}$ means that the sequence $\Gamma_{n}\left(x^{*}, y^{*}\right)$ is non-increasing and lower bounded by 0 . Then the sequence $\Gamma_{n}\left(x^{*}, y^{*}\right)$ converges to a finite limit $l\left(x^{*}, y^{*}\right)$. Thus, $\left\{x_{n}\right\}$ and $\left\{y_{n}\right\}$ are bounded. Moreover, passing to the limit in (3.6), we get

$$
\lim _{n \rightarrow \infty}\left\|A x_{n}-B y_{n}\right\|=0 \text {. }
$$

Since $t_{n}=J_{E_{1}^{*}}^{q}\left(J_{E_{1}}^{p}\left(x_{n}\right)-\gamma_{n} A^{*} J_{E_{3}}^{p}\left(A x_{n}-B y_{n}\right)\right)$, we deduce

$$
\begin{aligned}
\left\|J_{\mathrm{E}_{1}}^{\mathrm{p}}\left(\mathrm{t}_{\mathrm{n}}\right)-\mathrm{J}_{\mathrm{E}_{1}}^{\mathrm{p}}\left(x_{n}\right)\right\| & =\left\|J_{\mathrm{E}_{1}}^{\mathrm{p}}\left(x_{n}\right)-\gamma_{n} A^{*} J_{\mathrm{E}_{3}}^{\mathrm{p}}\left(A x_{n}-B y_{n}\right)-J_{\mathrm{E}_{1}}^{\mathrm{p}}\left(x_{n}\right)\right\| \\
& =\left\|-\gamma_{n} A^{*} J_{\mathrm{E}_{3}}^{\mathrm{p}}\left(A x_{n}-B y_{n}\right)\right\| \\
& \leqslant \gamma_{n}\|A\|\left\|J_{\mathrm{E}_{3}}^{\mathrm{p}}\left(A x_{n}-B y_{n}\right)\right\| \\
& \leqslant\left(\frac{q}{C_{q}\left(\|A\|^{q}+\|B\| q\right)}\right)^{\frac{1}{q-1}}\|A\|\left\|J_{\mathrm{E}_{3}}^{\mathrm{p}}\left(A x_{n}-B y_{n}\right)\right\| \\
& =\left(\frac{q}{C_{q}\left(\|A\|^{q}+\|B\| q\right)}\right)^{\frac{1}{q-1}}\|A\|\left\|A x_{n}-B y_{n}\right\|^{p-1} .
\end{aligned}
$$


Then, (3.7) yields that

$$
\lim _{n \rightarrow \infty}\left\|J_{E_{1}}^{p}\left(t_{n}\right)-J_{E_{1}}^{p}\left(x_{n}\right)\right\|=0 .
$$

It follows from the fact that $\mathrm{J}_{\mathrm{E}_{1}}^{\mathrm{p}}$ is norm-to-norm uniformly continuous that

$$
\lim _{n \rightarrow \infty}\left\|t_{n}-x_{n}\right\|=0 .
$$

Similarly, by $w_{n}=J_{E_{2}^{*}}^{q}\left(J_{E_{2}}^{p}\left(y_{n}\right)+\gamma_{n} B^{*} J_{E_{3}}^{p}\left(A x_{n}-B y_{n}\right)\right)$, then

$$
\begin{aligned}
\left\|J_{\mathrm{E}_{2}}^{\mathrm{p}}\left(w_{n}\right)-\mathrm{J}_{\mathrm{E}_{2}}^{\mathrm{p}}\left(y_{n}\right)\right\| & =\left\|J_{\mathrm{E}_{2}}^{\mathrm{p}}\left(y_{n}\right)+\gamma_{n} B^{*} J_{\mathrm{E}_{3}}^{\mathrm{p}}\left(A x_{n}-B y_{n}\right)-J_{\mathrm{E}_{2}}^{\mathrm{p}}\left(y_{n}\right)\right\| \\
& =\left\|\gamma_{n} \mathrm{~B}^{*} J_{\mathrm{E}_{3}}^{\mathrm{p}}\left(A x_{n}-B y_{n}\right)\right\| \\
& \leqslant \gamma_{n}\|B\|\left\|J_{\mathrm{E}_{3}}^{\mathrm{p}}\left(A x_{n}-B y_{n}\right)\right\| \\
& \leqslant\left(\frac{q}{C_{q}\left(\|A\|^{q}+\|B\|^{q}\right)}\right)^{\frac{1}{q-1}}\|B\|\left\|J_{E_{3}}^{p}\left(A x_{n}-B y_{n}\right)\right\| \\
& =\left(\frac{q}{C_{q}\left(\|A\|^{q}+\|B\|^{q}\right)}\right)^{\frac{1}{q-1}}\|B\|\left\|A x_{n}-B y_{n}\right\|^{p-1} .
\end{aligned}
$$

Hence, (3.7) leads to

$$
\lim _{n \rightarrow \infty}\left\|J_{E_{1}}^{p}\left(w_{n}\right)-J_{E_{1}}^{p}\left(y_{n}\right)\right\|=0 .
$$

The fact that $\mathrm{J}_{\mathrm{E}_{2}}^{\mathrm{p}}$ is norm-to-norm uniformly continuous can ensure

$$
\lim _{n \rightarrow \infty}\left\|w_{n}-y_{n}\right\|=0
$$

From (2.3), (3.1), (3.2), (3.3), and (3.4), we have

$$
\begin{aligned}
\Delta_{\mathfrak{p}}\left(t_{n}, u_{n}\right)= & \Delta_{p}\left(t_{n}, \Pi_{C} t_{n}\right) \\
\leqslant & \Delta_{p}\left(t_{n}, x^{*}\right)-\Delta_{p}\left(u_{n}, x^{*}\right) \\
\leqslant & \Delta_{p}\left(x_{n}, x^{*}\right)-\gamma_{n}\left\langle J_{E_{3}}^{p}\left(A x_{n}-B y_{n}\right), A x_{n}-A x^{*}\right\rangle \\
& +\frac{C_{q}\left(\gamma_{n}\|A\|\right)^{q}}{q}\left\|A x_{n}-B y_{n}\right\|^{p}-\Delta_{p}\left(x_{n+1}, x^{*}\right),
\end{aligned}
$$

and

$$
\begin{aligned}
& \Delta_{\mathfrak{p}}\left(w_{n}, v_{n}\right)=\Delta_{\mathfrak{p}}\left(w_{n}, \Pi_{\mathrm{Q}} w_{n}\right) \\
& \leqslant \Delta_{\mathfrak{p}}\left(w_{n}, \mathrm{y}^{*}\right)-\Delta_{\mathfrak{p}}\left(v_{n}, \mathrm{y}^{*}\right) \\
& \leqslant \Delta_{p}\left(y_{n}, y^{*}\right)-\gamma_{n}\left\langle J_{E_{3}}^{p}\left(A x_{n}-B y_{n}\right), B y^{*}-B y_{n}\right\rangle \\
& +\frac{C_{q}\left(\gamma_{n}\|B\|\right)^{q}}{q}\left\|A x_{n}-B y_{n}\right\|^{p}-\Delta_{p}\left(y_{n+1}, y^{*}\right) \text {. }
\end{aligned}
$$

Adding (3.10) and (3.11), we obtain

$$
\begin{aligned}
\Delta_{\mathfrak{p}}\left(t_{n}, u_{n}\right)+\Delta_{p}\left(w_{n}, v_{n}\right) \leqslant & \Delta_{p}\left(x_{n}, x^{*}\right)+\Delta_{p}\left(y_{n}, y^{*}\right)-\left(\Delta_{p}\left(x_{n+1}, x^{*}\right)+\Delta_{p}\left(y_{n+1}, y^{*}\right)\right) \\
& -\left(\gamma_{n}-\frac{C_{q}\left(\gamma_{n}\|A\|\right)^{q}+\left(\gamma_{n}\|B\|\right)^{q}}{q}\right)\left\|A x_{n}-B y_{n}\right\|^{p} .
\end{aligned}
$$

It follows that

$$
\lim _{n \rightarrow \infty}\left\|t_{n}-u_{n}\right\|=0,
$$

and

$$
\lim _{n \rightarrow \infty}\left\|w_{n}-v_{n}\right\|=0,
$$


which, with (3.8) and (3.9), yields

$$
\lim _{n \rightarrow \infty}\left\|x_{n}-u_{n}\right\|=0
$$

and

$$
\lim _{n \rightarrow \infty}\left\|y_{n}-v_{n}\right\|=0 \text {. }
$$

On the other hand, we show that $\lim _{n \rightarrow \infty}\left\|u_{n}-T u_{n}\right\|=0$, and $\lim _{n \rightarrow \infty}\left\|v_{n}-U v_{n}\right\|=0$. In fact, from (3.1) and (3.3), one has

$$
\begin{aligned}
0 \leqslant & \Delta_{\mathfrak{p}}\left(u_{n}, x^{*}\right)-\Delta_{\mathfrak{p}}\left(T u_{n}, x^{*}\right) \\
= & \Delta_{\mathfrak{p}}\left(u_{n}, x^{*}\right)-\Delta_{\mathfrak{p}}\left(x_{n+1}, x^{*}\right)+\Delta_{p}\left(x_{n+1}, x^{*}\right)-\Delta_{p}\left(T u_{n}, x^{*}\right) \\
\leqslant & \Delta_{p}\left(x_{n}, x^{*}\right)-\gamma_{n}\left\langle J_{E_{3}}^{p}\left(A x_{n}-B y_{n}\right), A x_{n}-A x^{*}\right\rangle+\frac{C_{q}\left(\gamma_{n}\|A\|\right)^{q}}{q}\left\|A x_{n}-B y_{n}\right\|^{p} \\
& -\Delta_{p}\left(x_{n+1}, x^{*}\right)+\alpha_{n} \Delta_{p}\left(u_{n}, x^{*}\right)+\left(1-\alpha_{n}\right) \Delta_{p}\left(T u_{n}, x^{*}\right)-\Delta_{p}\left(T u_{n}, x^{*}\right) \\
\leqslant & \Delta_{p}\left(x_{n}, x^{*}\right)-\gamma_{n}\left\langle J_{E_{3}}^{p}\left(A x_{n}-B y_{n}\right), A x_{n}-A x^{*}\right\rangle+\frac{C_{q}\left(\gamma_{n}\|A\|\right)^{q}}{q}\left\|A x_{n}-B y_{n}\right\|^{p} \\
& -\Delta_{p}\left(x_{n+1}, x^{*}\right)+\alpha_{n}\left(\Delta_{p}\left(u_{n}, x^{*}\right)-\Delta_{p}\left(T u_{n}, x^{*}\right)\right),
\end{aligned}
$$

which is simplified to

$$
\begin{aligned}
0 \leqslant & \left(1-\alpha_{n}\right)\left(\Delta_{p}\left(u_{n}, x^{*}\right)-\Delta_{p}\left(T u_{n}, x^{*}\right)\right) \\
\leqslant & \Delta_{p}\left(x_{n}, x^{*}\right)-\gamma_{n}\left\langle J_{E_{3}}^{p}\left(A x_{n}-B y_{n}\right), A x_{n}-A x^{*}\right\rangle \\
& +\frac{C_{q}\left(\gamma_{n}\|A\|\right)^{q}}{q}\left\|A x_{n}-B y_{n}\right\|^{p}-\Delta_{p}\left(x_{n+1}, x^{*}\right)
\end{aligned}
$$

Similarly, one has

$$
\begin{aligned}
& 0 \leqslant \Delta_{\mathrm{p}}\left(v_{\mathrm{n}}, \mathrm{y}^{*}\right)-\Delta_{\mathrm{p}}\left(\mathrm{U} v_{\mathrm{n}}, \mathrm{y}^{*}\right) \\
& =\Delta_{p}\left(v_{n}, y^{*}\right)-\Delta_{p}\left(y_{n+1}, y^{*}\right)+\Delta_{p}\left(y_{n+1}, y^{*}\right)-\Delta_{p}\left(U v_{n}, y^{*}\right) \\
& \leqslant \Delta_{\mathrm{p}}\left(\mathrm{y}_{n}, \mathrm{y}^{*}\right)-\gamma_{n}\left\langle\mathrm{~J}_{\mathrm{E}_{3}}^{\mathrm{p}}\left(A x_{n}-B y_{n}\right), B y^{*}-B y_{n}\right\rangle+\frac{C_{q}\left(\gamma_{n}\|B\|\right)^{q}}{q}\left\|A x_{n}-B y_{n}\right\|^{p} \\
& -\Delta_{p}\left(y_{n+1}, y^{*}\right)+\alpha_{n} \Delta_{p}\left(v_{n}, y^{*}\right)+\left(1-\alpha_{n}\right) \Delta_{p}\left(U v_{n}, y^{*}\right)-\Delta_{p}\left(U v_{n}, y^{*}\right) \\
& \leqslant \Delta_{\mathrm{p}}\left(\mathrm{y}_{n}, \mathrm{y}^{*}\right)-\gamma_{n}\left\langle\mathrm{~J}_{\mathrm{E}_{3}}^{\mathrm{p}}\left(A x_{n}-B y_{n}\right), B y^{*}-B y_{n}\right\rangle+\frac{C_{\mathrm{q}}\left(\gamma_{n}\|\mathrm{~B}\|\right)^{\mathrm{q}}}{\mathrm{q}}\left\|A x_{n}-B y_{n}\right\|^{\mathrm{p}} \\
& -\Delta_{\mathfrak{p}}\left(\mathrm{y}_{\mathfrak{n}+1}, \mathrm{y}^{*}\right)+\alpha_{\mathfrak{n}}\left(\Delta_{\mathfrak{p}}\left(v_{n}, \mathrm{y}^{*}\right)-\Delta_{\mathfrak{p}}\left(\mathrm{U} v_{\mathfrak{n}}, \mathrm{y}^{*}\right)\right) \text {, }
\end{aligned}
$$

which implies that

$$
\begin{aligned}
0 \leqslant & \left(1-\alpha_{n}\right)\left(\Delta_{p}\left(v_{n}, y^{*}\right)-\Delta_{p}\left(U v_{n}, y^{*}\right)\right) \\
\leqslant & \Delta_{p}\left(y_{n}, y^{*}\right)-\gamma_{n}\left\langle J_{E_{3}}^{p}\left(A x_{n}-B y_{n}\right), B y^{*}-B y_{n}\right\rangle \\
& +\frac{C_{q}\left(\gamma_{n}\|B\|\right)^{q}}{q}\left\|A x_{n}-B y_{n}\right\|^{p}-\Delta_{p}\left(y_{n+1}, y^{*}\right)
\end{aligned}
$$

Hence, (3.14) and (3.15) reduce to

$$
\begin{aligned}
0 \leqslant & \left(1-\alpha_{n}\right)\left[\left(\Delta_{p}\left(u_{n}, x^{*}\right)-\Delta_{p}\left(T u_{n}, x^{*}\right)\right)+\left(\Delta_{p}\left(v_{n}, y^{*}\right)-\Delta_{p}\left(U v_{n}, y^{*}\right)\right)\right] \\
\leqslant & \Delta_{p}\left(x_{n}, x^{*}\right)+\Delta_{p}\left(y_{n}, y^{*}\right)-\left(\Delta_{p}\left(x_{n+1}, x^{*}\right)+\Delta_{p}\left(y_{n+1}, y^{*}\right)\right) \\
& -\left(\gamma_{n}-\frac{C_{q}\left(\left(\gamma_{n}\|A\|\right)^{q}+\left(\gamma_{n}\|B\|\right)^{q}\right)}{q}\right)\left\|A x_{n}-B y_{n}\right\|^{p} .
\end{aligned}
$$


It follows that

$$
\lim _{n \rightarrow \infty}\left(\Delta_{\mathfrak{p}}\left(u_{n}, x^{*}\right)-\Delta_{p}\left(T u_{n}, x^{*}\right)\right)=0
$$

and

$$
\lim _{n \rightarrow \infty}\left(\Delta_{p}\left(v_{n}, y^{*}\right)-\Delta_{p}\left(U v_{n}, y^{*}\right)\right)=0
$$

From the fact that $\mathrm{T}$ and $\mathrm{U}$ are left Bregman strongly nonexpansive mappings, we have

$$
\lim _{n \rightarrow \infty} \Delta_{\mathfrak{p}}\left(u_{n}, T u_{n}\right)=0, \quad \text { and } \quad \lim _{n \rightarrow \infty} \Delta_{p}\left(v_{n}, U v_{n}\right)=0
$$

Thus, we obtain

$$
\lim _{n \rightarrow \infty}\left\|u_{n}-T u_{n}\right\|=0
$$

and

$$
\lim _{n \rightarrow \infty}\left\|v_{n}-u v_{n}\right\|=0 .
$$

In what follows, we will prove that $\left\{x_{n}\right\}$ and $\left\{y_{n}\right\}$ are asymptotically regular. Indeed, it follows from (1.6) that

$$
\left\|\alpha_{n} J_{E_{1}}^{p}\left(u_{n}\right)+\left(1-\alpha_{n}\right) J_{E_{1}}^{p}\left(T u_{n}\right)-J_{E_{1}}^{p}\left(u_{n}\right)\right\|=\left(1-\alpha_{n}\right)\left\|J_{E_{1}}^{p}\left(T u_{n}\right)-J_{E_{1}}^{p}\left(u_{n}\right)\right\|,
$$

and

$$
\left\|\alpha_{n} J_{E_{2}}^{p}\left(v_{n}\right)+\left(1-\alpha_{n}\right) J_{E_{2}}^{p}\left(U u_{n}\right)-J_{E_{2}}^{p}\left(v_{n}\right)\right\|=\left(1-\alpha_{n}\right)\left\|J_{E_{2}}^{p}\left(U v_{n}\right)-J_{E_{2}}^{p}\left(v_{n}\right)\right\| .
$$

Since $\mathrm{J}_{\mathrm{E}_{1}^{*}}^{\mathrm{q}}$ and $\mathrm{J}_{\mathrm{E}_{2}^{*}}^{\mathrm{q}}$ are norm-to-norm uniformly continuous, we have

$$
\lim _{n \rightarrow \infty}\left\|\alpha_{n} J_{E_{1}}^{p}\left(u_{n}\right)+\left(1-\alpha_{n}\right) J_{E_{1}}^{p}\left(T u_{n}\right)-J_{E_{1}}^{p}\left(u_{n}\right)\right\|=0,
$$

and

$$
\lim _{n \rightarrow \infty}\left\|\alpha_{n} J_{E_{2}}^{p}\left(v_{n}\right)+\left(1-\alpha_{n}\right) J_{E_{2}}^{p}\left(U v_{n}\right)-J_{E_{2}}^{p}\left(v_{n}\right)\right\|=0
$$

Consequently,

$$
\lim _{n \rightarrow \infty}\left\|x_{n+1}-u_{n}\right\|=0
$$

and

$$
\lim _{n \rightarrow \infty}\left\|y_{n+1}-v_{n}\right\|=0
$$

which, with (3.12) and (3.13), yields

$$
\lim _{n \rightarrow \infty}\left\|x_{n+1}-x_{n}\right\|=0
$$

and

$$
\lim _{n \rightarrow \infty}\left\|y_{n+1}-y_{n}\right\|=0 .
$$

Next, we prove that $(x, y) \in \Omega$. Since $\left\{x_{n}\right\}$ is bounded, there exists $\left\{x_{n_{j}}\right\}$ of $\left\{x_{n}\right\}$ such that $x_{n_{j}} \rightarrow x \in$ $w_{w}\left(x_{n}\right)$. Now, from $x_{n_{j}} \rightarrow x$ and (3.12), we deduce that $u_{n_{j}} \rightarrow x$. Due to (2.2), we have

$$
\begin{aligned}
\Delta_{\mathfrak{p}}\left(x, \Pi_{\mathrm{C}} x\right) & \leqslant\left\langle\mathrm{J}_{\mathrm{E}_{1}}^{\mathrm{p}}(x)-\mathrm{J}_{\mathrm{E}_{1}}^{\mathrm{p}}\left(\Pi_{\mathrm{C}} x\right), x-\Pi_{\mathrm{C}} x\right\rangle \\
& =\left\langle\mathrm{J}_{\mathrm{E}_{1}}^{\mathrm{p}}(x)-\mathrm{J}_{\mathrm{E}_{1}}^{\mathrm{p}}\left(\Pi_{\mathrm{C}} x\right), x-\mathrm{u}_{n_{j}}\right\rangle+\left\langle\mathrm{J}_{\mathrm{E}_{1}}^{\mathrm{p}}(x)-\mathrm{J}_{\mathrm{E}_{1}}^{\mathrm{p}}\left(\Pi_{\mathrm{C}} x\right), u_{n_{j}}-\Pi_{\mathrm{C}} x\right\rangle \\
& \leqslant\left\langle J_{\mathrm{E}_{1}}^{\mathrm{p}}(x)-\mathrm{J}_{\mathrm{E}_{1}}^{\mathrm{p}}\left(\Pi_{\mathrm{C}} x\right), x-\mathrm{u}_{n_{\mathrm{j}}}\right\rangle .
\end{aligned}
$$

As $j \rightarrow \infty$, one has that $\Delta_{p}\left(x, \Pi_{C} x\right)=0$ which means $x \in C$. Furthermore, (3.16) reveals that $x$ is an asymptotic fixed point and then $x \in F(\hat{T})=F(T)$. Thus, $x \in C \cap F(T)$. Since $\left\{y_{n}\right\}$ is bounded, there exists $\left\{y_{n_{j}}\right\}$ of $\left\{y_{n}\right\}$ such that $y_{n_{j}} \rightarrow y \in w_{w}\left(y_{n}\right)$. Similarly, we have $y \in Q \cap F(U)$. Furthermore, it follows from (3.7) that $\|A x-B y\| \leqslant \lim _{\inf _{k \rightarrow \infty}}\left\|A x_{n}-B y_{n}\right\|=0$, which means that $(x, y) \in \Omega$. 
Finally, we will reveal the uniqueness of the weak cluster points of $\left\{x_{n}\right\}$ and $\left\{y_{n}\right\}$. In fact, assume that $\bar{x}^{*}$ and $\bar{y}^{*}$ are another weak cluster points of $\left\{x_{n}\right\}$ and $\left\{y_{n}\right\}$. From the definition of $\Gamma_{n}$, we deduce

$$
\begin{aligned}
& \Gamma_{\mathrm{n}}(\mathrm{x}, \mathrm{y})=\Delta_{\mathrm{p}}\left(\mathrm{x}_{\mathrm{n}}, \mathrm{x}\right)+\Delta_{\mathrm{p}}\left(\mathrm{y}_{\mathrm{n}}, \mathrm{y}\right) \\
& =\Delta_{\mathrm{p}}\left(\mathrm{x}_{\mathrm{n}}, \overline{\mathrm{x}}^{*}\right)+\Delta_{\mathrm{p}}\left(\bar{x}^{*}, \mathrm{x}\right)+\left\langle\bar{x}^{*}-\mathrm{x}, \mathrm{J}_{\mathrm{E}_{1}}^{\mathrm{p}} \mathrm{x}_{\mathrm{n}}-\mathrm{J}_{\mathrm{E}_{1}}^{\mathrm{p}} \overline{\mathrm{x}}^{*}\right\rangle \\
& +\Delta_{p}\left(y_{n}, \bar{y}^{*}\right)+\Delta_{p}\left(\bar{y}^{*}, y\right)+\left\langle\bar{y}^{*}-y, J_{E_{2}}^{p} y_{n}-J_{E_{2}}^{p} \bar{y}^{*}\right\rangle \\
& =\Delta_{p}\left(x_{n}, \bar{x}^{*}\right)+\Delta_{p}\left(y_{n}, \bar{y}^{*}\right)+\Delta_{p}\left(\bar{x}^{*}, x\right)+\Delta_{p}\left(\bar{y}^{*}, y\right) \\
& +\left\langle\bar{x}^{*}-x, J_{\mathrm{E}_{1}}^{\mathrm{p}} x_{n}-\mathrm{J}_{\mathrm{E}_{1}}^{\mathrm{p}} \bar{x}^{*}\right\rangle+\left\langle\overline{\mathrm{y}}^{*}-\mathrm{y}_{1} \mathrm{~J}_{\mathrm{E}_{2}}^{\mathrm{p}} \mathrm{y}_{\mathrm{n}}-\mathrm{J}_{\mathrm{E}_{2}}^{\mathrm{p}} \overline{\mathrm{y}}^{*}\right\rangle \text {. }
\end{aligned}
$$

Without loss of generality, assume that $x_{n} \rightarrow \bar{x}^{*}$ and $y_{n} \rightarrow \bar{y}^{*}$, and $J_{E_{2}}^{p}$ and $J_{E_{2}}^{p}$ are weak-to-weakcontinuous, then

$$
l(x, y)=l\left(\bar{x}^{*}, \bar{y}^{*}\right)+\Delta_{p}\left(\bar{x}^{*}, x\right)+\Delta_{p}\left(\bar{y}^{*}, y\right) .
$$

Reversing the role of $(x, y)$ and $\left(\bar{x}^{*}, \bar{y}^{*}\right)$, one has

$$
l\left(\bar{x}^{*}, \bar{y}^{*}\right)=l(x, y)+\Delta_{p}\left(x, \bar{x}^{*}\right)+\Delta_{p}\left(y, \bar{y}^{*}\right) .
$$

Thanking to (2.1), (3.17), and (3.18), we obtain

$$
\begin{aligned}
\tau\left(\left\|\bar{x}^{*}-x\right\|^{\mathrm{p}}+\left\|\overline{\mathrm{y}}^{*}-\mathrm{y}\right\|^{\mathrm{p}}\right) & \leqslant \Delta_{\mathrm{p}}\left(\overline{\mathrm{x}}^{*}, x\right)+\Delta_{\mathfrak{p}}\left(x, \bar{x}^{*}\right)+\Delta_{\mathfrak{p}}\left(\mathrm{y}, \overline{\mathrm{y}}^{*}\right)+\Delta_{\mathfrak{p}}\left(\overline{\mathrm{y}}^{*}, \mathrm{y}\right) \\
& =\left\langle\overline{\mathrm{x}}^{*}-x, \mathrm{~J}_{\mathrm{E}_{1}}^{\mathrm{p}} \overline{\mathrm{x}}^{*}-\mathrm{J}_{\mathrm{E}_{1}}^{\mathrm{p}} \mathrm{x}\right\rangle+\left\langle\overline{\mathrm{y}}^{*}-\mathrm{y}, \mathrm{J}_{2} \overline{\mathrm{y}}^{\mathrm{p}}-\mathrm{J}_{\mathrm{E}_{2}}^{\mathrm{p}} \mathrm{y}\right\rangle=0,
\end{aligned}
$$

which yields that $\bar{x}^{*}=x$, and $\bar{y}^{*}=y$. Hence, the sequence $\left(x_{n}, y_{n}\right)$ weakly converges to a solution of the problem (1.5), which completes the proof.

\section{Application}

We now turn to apply our iterative scheme to approximate a solution of the split feasibility problem in the framework of p-uniformly convex and uniformly smooth Banach spaces.

Consider the split feasibility problem:

$$
x \in C \text {, such that } A x \in Q \text {, }
$$

where $E_{1}$ and $E_{2}$ are Hilbert spaces, and $C \subset E_{1}$ and $Q \subset E_{2}$ are nonempty, closed, and convex sets. $A: E_{1} \rightarrow E_{2}$ is a bounded linear operator. The split feasibility problem (4.1) was first introduced by Censor and Elfving [6] for modeling inverse problems which arise from phase retrievals and in medical image reconstruction [4]. For solving the split feasibility problem, Censor and Elfving [6] suggested the following scheme

$$
x_{k+1}=P_{C}\left(x_{k}-\lambda A^{*}\left(I-P_{Q}\right) A x_{k}\right),
$$

where $\lambda \in\left(0, \frac{2}{\gamma}\right)$, and $\gamma$ is the spectral radius of the operator $A^{*} A$. The split feasibility problem (4.1) was studied by Schöpfer et al. [24] when $E_{1}$ and $E_{2}$ are p-uniformly convex and uniformly smooth Banach spaces. They proposed the following algorithm

$$
x_{k+1}=\Pi_{C} J_{E_{1}^{*}}^{q}\left(J_{E_{1}}^{p}\left(x_{n}\right)-t A^{*} J_{E_{2}}^{p}\left(A x_{n}-P_{Q}\left(A x_{n}\right)\right)\right) .
$$

In what follows, we introduce an algorithm to solve the split feasibility problem, and fixed point problem for left Bregman strongly nonexpansive mapping in the framework of $p$-uniformly convex and uniformly smooth Banach spaces. 
Theorem 4.1. Let $\mathrm{E}_{1}$ and $\mathrm{E}_{2}$ be two p-uniformly convex and uniformly smooth real Banach spaces. Let $\mathrm{A}: \mathrm{E}_{1} \rightarrow \mathrm{E}_{3}$ be a bounded linear operator, and $\mathrm{A}^{*}: \mathrm{E}_{2}^{*} \rightarrow \mathrm{E}_{1}^{*}$ be the adjoint of $\mathrm{A}$. Let $\mathrm{T}$ be a left Bregman strongly nonexpansive mapping of $\mathrm{C}$ into $\mathrm{C}$ such that $\mathrm{F}(\mathrm{T})=\mathrm{F}(\hat{\mathrm{T}})$, and $\mathrm{U}$ be a left Bregman strongly nonexpansive mapping of $\mathrm{Q}$ into $\mathrm{Q}$ such that $\mathrm{F}(\mathrm{U})=\mathrm{F}(\hat{\mathrm{U}})$, where $\mathrm{C} \subset \mathrm{E}_{1}$ and $\mathrm{Q} \subset \mathrm{E}_{2}$ are nonempty, closed, and convex sets. For any initial guess $\left(x_{1}, y_{1}\right) \in E_{1} \times E_{2}$, define $\left(x_{n+1}, y_{n+1}\right)$ recursively by the following formula

$$
\left\{\begin{array}{l}
u_{n}=\Pi_{C} J_{E_{1}^{*}}^{q}\left(J_{E_{1}}^{p}\left(x_{n}\right)-\gamma_{n} A^{*} J_{E_{2}}^{p}\left(A x_{n}-y_{n}\right)\right), \\
x_{n+1}=J_{E_{1}^{*}}^{q}\left(\alpha_{n} J_{E_{1}}^{p}\left(u_{n}\right)+\left(1-\alpha_{n}\right) J_{E_{1}}^{p}\left(T u_{n}\right)\right), \\
v_{n}=\Pi_{Q} J_{E_{2}^{*}}^{q}\left(J_{E_{2}}^{p}\left(y_{n}\right)+\gamma_{n} J_{E_{2}}^{p}\left(A x_{n}-y_{n}\right)\right), \\
y_{n+1}=J_{E_{2}^{*}}^{q}\left(\alpha_{n} J_{E_{2}}^{p}\left(v_{n}\right)+\left(1-\alpha_{n}\right) J_{E_{2}}^{p}\left(U v_{n}\right)\right),
\end{array}\right.
$$

where $\left\{\alpha_{n}\right\}$ is a sequence in $(0,1)$ and the sequence $\left\{\gamma_{n}\right\}$ satisfies $0<\mathrm{a}_{1}<\gamma_{n}<\mathrm{a}_{2}<\left(\frac{\mathrm{q}}{\mathrm{C}_{\mathrm{q}}\left(\|\mathrm{A}\|\left\|^{\mathrm{q}}+\right\| \mathrm{I} \| \mathrm{q}\right)}\right)^{\frac{1}{\mathrm{q}-1}}$. If the solution set $\Omega$ of (4.1) is nonempty, then the sequence $\left(x_{n}, y_{n}\right)$ generated by (4.2) weakly converges to a solution $(x, y)$ of (4.1). Furthermore, $\left\|A x_{n}-y_{n}\right\| \rightarrow 0,\left\|x_{n}-x_{n+1}\right\| \rightarrow 0$, and $\left\|y_{n}-y_{n+1}\right\| \rightarrow 0$ as $n \rightarrow \infty$.

Proof. Let $\mathrm{E}_{2}=\mathrm{E}_{3}, \mathrm{~B}=\mathrm{I}, \mathrm{F}(\mathrm{T})=\mathrm{C}, \mathrm{F}(\mathrm{U})=\mathrm{Q}$ in (1.5), then Theorem 3.1 reduces the desired conclusion.

\section{Numerical examples}

In this section, we present some simple numerical examples to confirm the feasibility of the proposed methods. All the numerical results are carried out on a personal Lenovo Thinkpad computer with Intel(R) Core(TM) i7-6500U CPU 2.50GHz and RAM 8.00GB. We denote the vector with all elements 1 by $e_{1}$.

Example 5.1. Let $E_{1}=E_{2}=E_{3}=H$, where $H$ is a real Hilbert space. Set

$$
\begin{aligned}
A & =\left(\begin{array}{llll}
0.935824215383956 & 0.0786227516093100 & 0.533090326961467 \\
0.481772301596827 & 0.445407925813256 & 0.206323180349956 \\
0.328475651526597 & 0.465510336176384 & 0.156967245259197 \\
0.803081686629851 & 0.736678637754769 & 0.402239519762668 \\
0.143465565201252 & 0.699037204178715 & 0.437575868338577
\end{array}\right), \\
B & =\left(\begin{array}{llll}
0.975063468894014 & 0.544162281779400 & 0.370152466633967 & 0.403116857984051 \\
0.926744192452944 & 0.842279598560883 & 0.436120802744759 & 0.0539818085177951 \\
0.308064151740705 & 0.787970869649794 & 0.633202504891210 & 0.135984719774865 \\
0.862582601622939 & 0.536371208102865 & 0.146777396544920 & 0.390509652996561 \\
0.560227281225362 & 0.00183038387212930 & 0.565367033193397 & 0.457555564498429
\end{array}\right),
\end{aligned}
$$

$C=\left\{x \in R^{3}:\|x\| \leqslant 2\right\}, Q=\left\{y \in R^{4}:-3 e_{1} \leqslant y \leqslant e_{1}\right\}$, and $\alpha_{n}=\frac{1}{8}+\frac{1}{2 n}$ in (1.6).

We take the following two cases as the initial value

Case one: $x_{1}=10 *$ ones $(3,1), y_{1}=10 *$ ones $(4,1)$.

Case two: $x_{1}=1 *$ ones $(3,1), y_{1}=1 *$ ones $(4,1)$.

In the implementation, we choose $\left\|A x_{n}-B y_{n}\right\| \leqslant 10^{-4}$ as the stopping criterion. Figs 1 and 2 reveal that the iteration process of the sequences $\left\|x_{n}\right\|,\left\|y_{n}\right\|$ and $\left\|A x_{n}-B y_{n}\right\|$ are monotone decreasing, and show that the more the iteration steps are, the more slowly the sequence converges. 

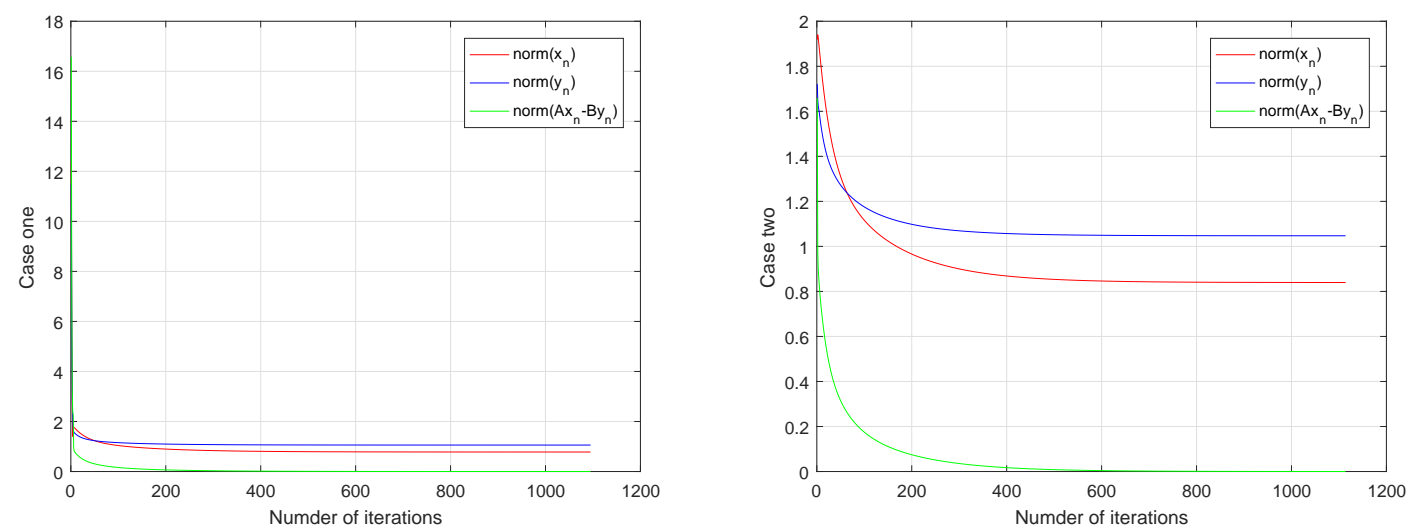

Figure 1: Behavior of $\left\|x_{n}\right\|,\left\|y_{n}\right\|$ and $\left\|A x_{n}-B y_{n}\right\|$ with $\gamma_{n}=0.8 * \frac{2}{\|A\|^{2}+\|B\|^{2}}$.
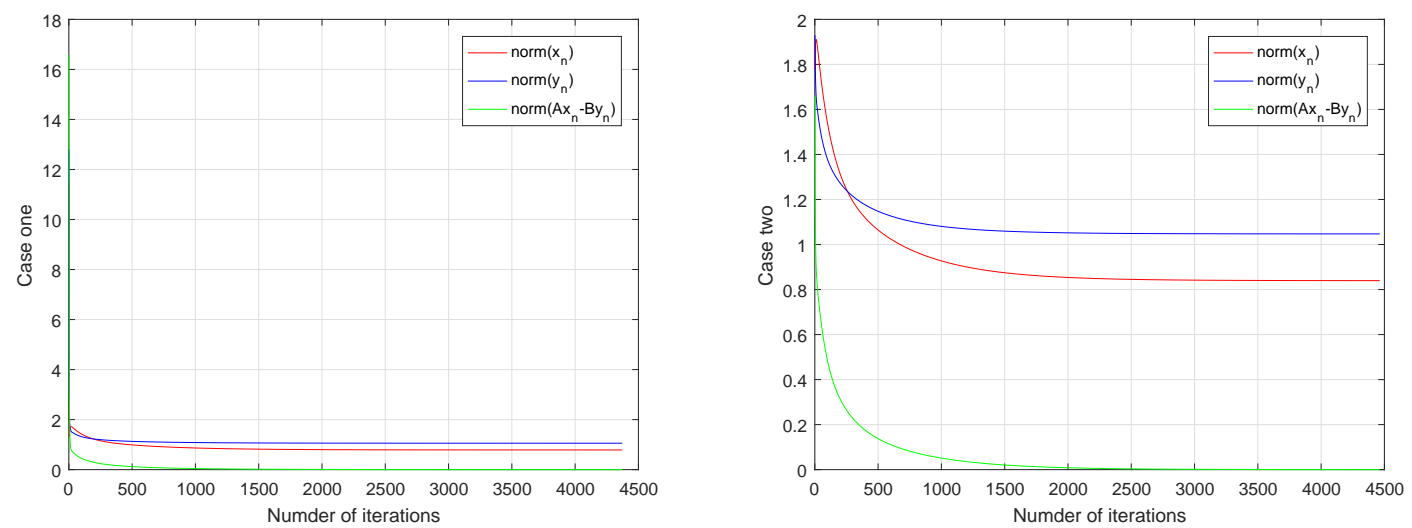

Figure 2: Behavior of $\left\|x_{n}\right\|,\left\|y_{n}\right\|$ and $\left\|A x_{n}-B y_{n}\right\|$ with $\gamma_{n}=0.2 * \frac{2}{\|A\|^{2}+\|B\|^{2}}$.

Example 5.2. Let $\mathrm{E}_{1}=\mathrm{E}_{2}=\mathrm{E}_{3}=\mathrm{L}_{2}([0,1])$ with the inner product

$$
\langle f, g\rangle=\int_{0}^{1} f(t) g(t) d t .
$$

Set

$$
C=\left\{x \in L_{2}([0,1]:\langle x, a\rangle=b\},\right.
$$

where $a=2 t^{2}, b=0$. Then

$$
\Pi_{C}(x)=P_{C}(x)=\max \left\{0, \frac{b-\langle a, x\rangle}{\|a\|^{2}}\right\} a+x .
$$

Set

$$
\mathrm{Q}=\left\{\mathrm{y} \in \mathrm{L}_{2}([0,1]:\langle\mathrm{y}, \mathrm{c}\rangle \geqslant \mathrm{d}\},\right.
$$

where $c=\frac{t}{3}, d=-1$. Then

$$
\Pi_{\mathrm{Q}}(\mathrm{x})=\mathrm{P}_{\mathrm{Q}}(\mathrm{x})=\frac{\mathrm{d}-\langle\mathrm{a}, \mathrm{y}\rangle}{\|\mathrm{c}\|^{2}} \mathrm{c}+\mathrm{y} .
$$

Define $A: L_{2}([0,1]) \rightarrow L_{2}([0,1])$ by $A x(t)=\frac{x(t)}{2}$, and $B: L_{2}([0,1]) \rightarrow L_{2}([0,1])$ by $B y(t)=\frac{y(t)}{4}$. Hence, $A$ and $B$ are bounded linear operators. Furthermore, from [14], we can choose $T=P_{C}$ and $U=P_{Q}$ and let $\alpha_{n}=\frac{1}{2}-\frac{1}{8 n}$. Then the problem (1.5) can reduce to

$$
x \in C, y \in Q \text {, such that } A x=B y .
$$

It is obvious that $(0,0) \in \Omega$ which means the solution set $\Omega$ is nonempty. Furthermore, the iterative 
scheme (1.6) can become

$$
\left\{\begin{array}{l}
u_{n}=P_{C}\left(x_{n}-\gamma_{n} A^{*}\left(A x_{n}-B y_{n}\right)\right) \\
x_{n+1}=\left(\frac{1}{2}-\frac{1}{8 n}\right) u_{n}+\left(\frac{1}{2}+\frac{1}{8 n}\right) P_{C}\left(u_{n}\right) \\
v_{n}=P_{Q}\left(y_{n}+\gamma_{n} B^{*}\left(A x_{n}-B y_{n}\right)\right) \\
y_{n+1}=\left(\frac{1}{2}-\frac{1}{8 n}\right) v_{n}+\left(\frac{1}{2}+\frac{1}{8 n}\right) P_{Q}\left(v_{n}\right)
\end{array}\right.
$$

We take

$$
\frac{\left\|x_{n}-x_{n-1}\right\|}{\left\|x_{2}-x_{1}\right\|}+\frac{\left\|y_{n}-y_{n-1}\right\|}{\left\|y_{2}-y_{1}\right\|} \leqslant 10^{-2}
$$

as the stopping criterion. Now, choose $\gamma_{n}=0.001$ and $\gamma_{n}=0.1$. Consider the following two cases.

Case one: if initial value $\left(x_{1}, y_{1}\right)=\left(3 \sin t, t^{2}\right)$, then the behaviors of $\left\|x_{n}-x_{n+1}\right\|$ and $\left\|y_{n}-y_{n+1}\right\|$ are presented respectively in Figs 3 and 4 . We have small reduction in the number of iterations when $\gamma_{n}$ is taken close to zero. The reason is worth for further research.

Case two: if initial value $\left(x_{1}, y_{1}\right)=\left(-e^{2 t}, 10 t\right)$, then the behaviors of $\left\|x_{n}-x_{n+1}\right\|$ and $\left\|y_{n}-y_{n+1}\right\|$ are presented respectively in Figs 5 and 6. We observe that the choice of different $\gamma_{n}$ has no effect on the convergent rate both in terms of the number of iterations obtained and the time taken.
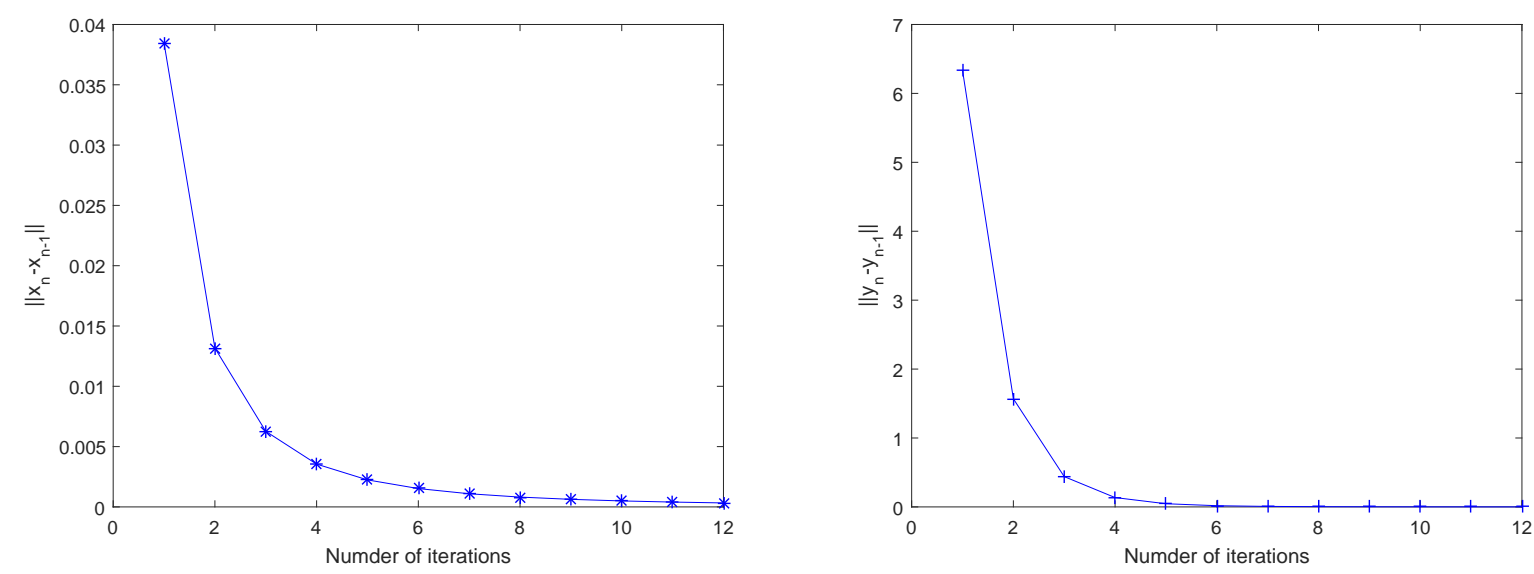

Figure 3: Behavior of $\left\|x_{n}-x_{n+1}\right\|$ and $\left\|y_{n}-y_{n+1}\right\|$ at the initial point $\left(3 \sin t, t^{2}\right)$ with $\gamma_{n}=0.001$.
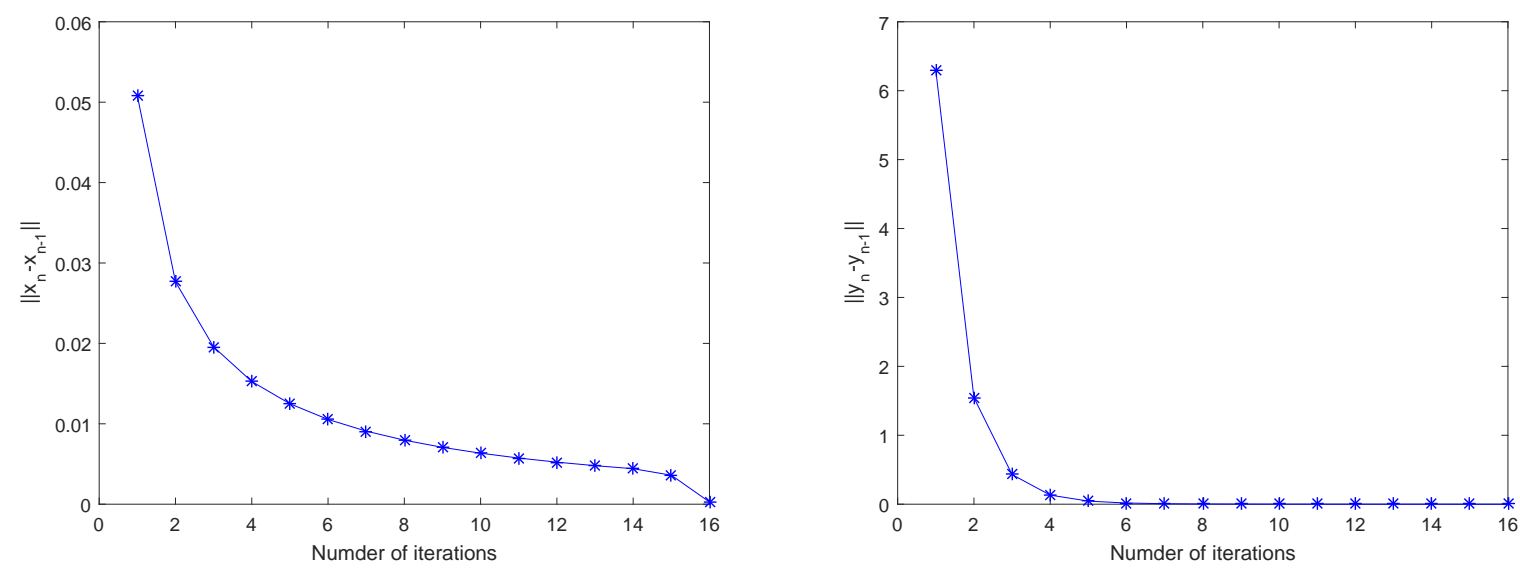

Figure 4: Behavior of $\left\|x_{n}-x_{n+1}\right\|$ and $\left\|y_{n}-y_{n+1}\right\|$ at the initial point $\left(3 \sin t, t^{2}\right)$ with $\gamma_{n}=0.1$. 

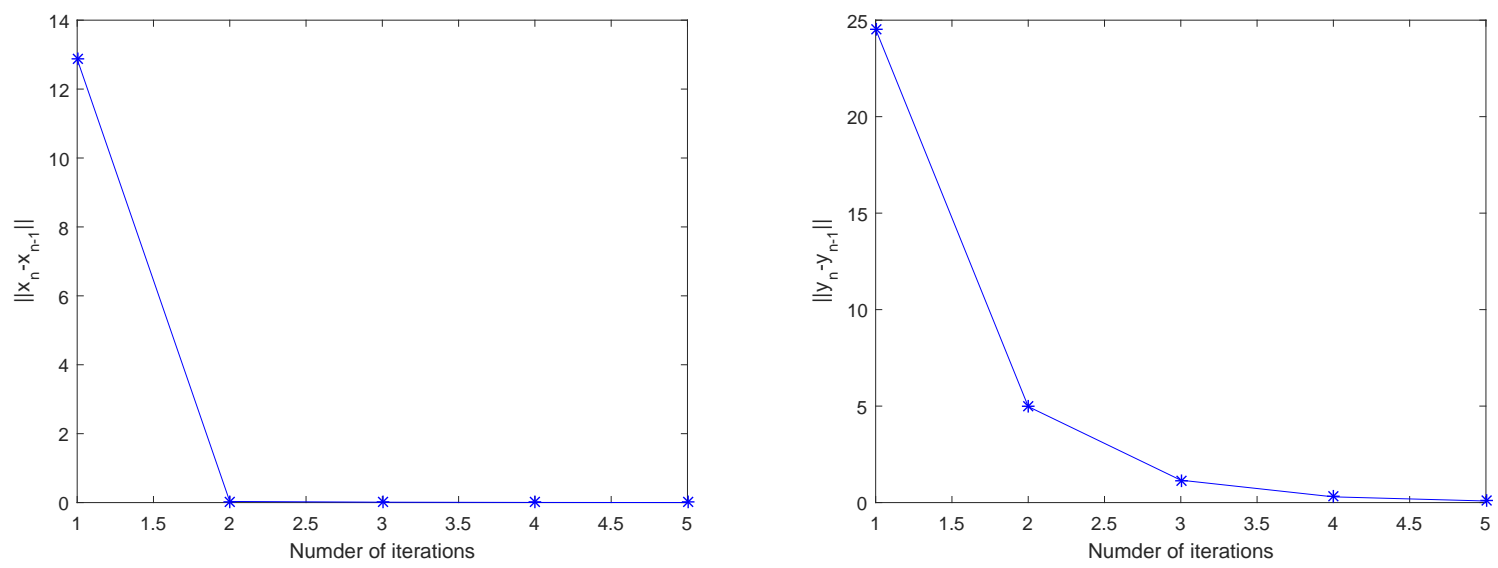

Figure 5: Behavior of $\left\|x_{n}-x_{n+1}\right\|$ and $\left\|y_{n}-y_{n+1}\right\|$ at the initial point $\left(-e^{2 t}, 10 t\right)$ with $\gamma_{n}=0.001$.
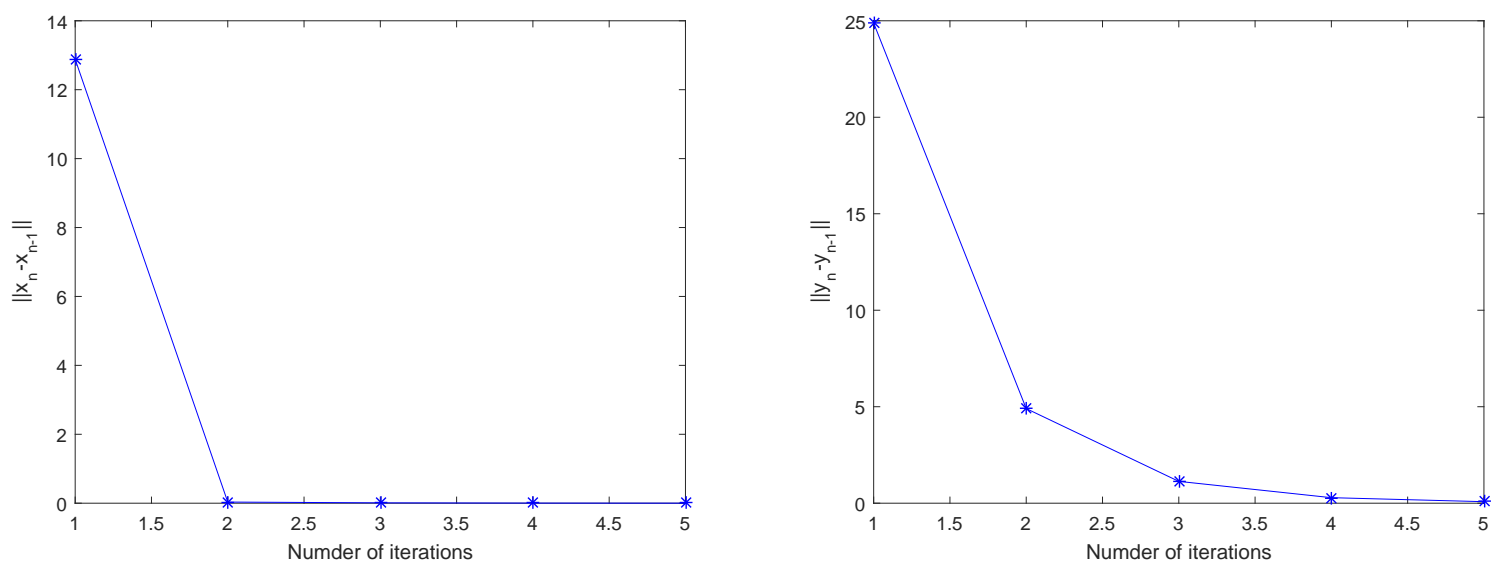

Figure 6: Behavior of $\left\|x_{n}-x_{n+1}\right\|$ and $\left\|y_{n}-y_{n+1}\right\|$ at the initial point $\left(-e^{2 t}, 10 t\right)$ with $\gamma_{n}=0.1$.

\section{Acknowledgment}

The authors were supported financially by the National Natural Science Foundation of China (11401438,11671228,11601261,11571120), Shandong Provincial Natural Science Foundation (ZR2016AQ12), and Project of Shandong Province Higher Educational Science and Technology Program (Grant No. J14LI52).

\section{References}

[1] Y. I. Alber, Metric and generalized projection operators in Banach spaces: properties and applications, Theory and applications of nonlinear operators of accretive and monotone type, Lecture Notes in Pure and Appl. Math., Dekker, New York, 178 (1996), 15-50. 2

[2] H. Attouch, J. Bolte, P. Redont, A. Soubeyran, Alternating proximal algorithms for weakly coupled convex minimization problems, Applications to dynamical games and PDE's, J. Convex Anal., 15 (2008), 485-506. 1

[3] H. Attouch, A. Cabot, F. Frankel, J. Peypouquet, Alternating proximal algorithms for linearly constrained variational inequalities: application to domain decomposition for PDE's, Nonlinear Anal., 74 (2011), 7455-7473. 1

[4] C. Byrne, Iterative oblique projection onto convex sets and the split feasibility problem, Inverse Problems, 18 (2002), 441-453. 4

[5] Y. Censor, T. Bortfeld, B. Martin, A. Trofimov, A unified approach for inversion problems in intensity-modulated radiation therapy, Phys. Med. Biol., 51 (2006), 2353-2365. 1

[6] Y. Censor, T. Elfving, A multiprojection algorithm using Bregman projections in a product space, Numer. Algorithms, 8 (1994), 221-239. 4

[7] Y. Censor, S. Reich, Iterations of paracontractions and firmly nonexpansive operators with applications to feasibility and optimization, Optimization, 37 (1996), 323-339. 2 
[8] H.-T. Che, M.-X. Li, A simultaneous iterative method for split equality problems of two finite families of strictly pseudononspreading mappings without prior knowledge of operator norms, Fixed Point Theory Appl., 2015 (2015), 14 pages. 1

[9] H.-T. Che , S.-J. Li, A damped simultaneous iterative method for split equality problems of asymptotically demi-contractive mapping in the intermediate sense without prior knowledge of operator norms, J. Interdiscip. Math., 19 (2016), 645-656.

[10] X.-N. Chi, H.-J. Wei, Y.-Q. Feng, J.-W. Chen, Variational inequality formulation of circular cone eigenvalue complementarity problems, Fixed Point Theory Appl., 2016 (2016), 14 pages. 1

[11] I. Cioranescu, Geometry of Banach spaces, duality mappings and nonlinear problems, Mathematics and its Applications, Kluwer Academic Publishers Group, Dordrecht, (1990). 2

[12] Q.-L. Dong, S.-N. He, J. Zhao, Solving the split equality problem without prior knowledge of operator norms, Optimization, 64 (2015), 1887-1906. 1

[13] J. Lindenstrauss, L. Tzafriri, Classical Banach spaces, II, Function spaces, Ergebnisse der Mathematik und ihrer Grenzgebiete [Results in Mathematics and Related Areas], Springer-Verlag, Berlin-New York, (1979). 2

[14] V. Martín-Márquez, S. Reich, S. Sabach, Bregman strongly nonexpansive operators in reflexive Banach spaces, J. Math. Anal. Appl., 400 (2013), 597-614. 2, 5.2

[15] A. Moudafi, A note on quasi split null-point feasibility problems, J. Nonlinear Anal. Optim., 5 (2013), 1-6. 1

[16] A. Moudafi, A relaxed alternating CQ-algorithm for convex feasibility problems, Nonlinear Anal., 79 (2013), 117-121. 1, 1

[17] A. Moudafi, Alternating CQ-algorithms for convex feasibility and split fixed-point problems, J. Nonlinear Convex Anal., 15 (2014), 809-818.

[18] A. Moudafi, E. Al-Shemas, Simultaneous iterative methods for split equality problem, Trans. Math. Program. Appl., 1 (2013), 1-11. 1

[19] B. Qu, N.-H. Xiu, A note on the CQ algorithm for the split feasibility problem, Inverse Problems, 21 (2005), $1655-1665$.

[20] B. Qu, N.-H. Xiu, A new halfspace-relaxation projection method for the split feasibility problem, Linear Algebra Appl., 5 (2008), 1218-1229. 1

[21] S. Reich, Book Review: Geometry of Banach spaces, duality mappings and nonlinear problems, Bull. Amer. Math. Soc. (N.S.), 26 (1992), 367-370. 2

[22] S. Reich, A weak convergence theorem for the alternating method with Bregman distances, Theory and applications of nonlinear operators of accretive and monotone type, Lecture Notes in Pure and Appl. Math., Dekker, New York, 178 (1996), 313-318. 2

[23] F. Schöpfer, Iterative regularization method for the solution of the split feasibility problem in Banach spaces, PhD thesis, Saarbrücken, Germany, (2007). 2

[24] F. Schöpfer, T. Schuster, A. Louis, An iterative regularization method for the solution of the split feasibility problem in Banach spaces, Inverse Problems, 24 (2008), 20 pages. 2, 4

[25] H. K. Xu, Inequalities in Banach spaces with applications, Nonlinear Anal., 16 (1991), 1127-1138. 2.1

[26] Y.-H. Yao, R. P. Agarwal, M. Postolache, Y.-C. Liou, Algorithms with strong convergence for the split common solution of the feasibility problem and fixed point problem, Fixed Point Theory Appl., 2014 (2014), 14 pages. 1

[27] Y.-H. Yao, Y.-C. Liou, J.-C. Yao, Split common fixed point problem for two quasi-pseudo-contractive operators and its algorithm construction, Fixed Point Theory Appl., 2015 (2015), 19 pages. 1, 2 